\title{
AB006. A modular analysis approach to studying gene expression in cutaneous lupus erythematosus
}

\section{Jane Zhu', Ly Tran' ${ }^{2}$ Frank Zheng' ${ }^{2}$ Judith James ${ }^{2}$, Joel Guthridge ${ }^{2}$, Benjamin Chong ${ }^{1}$}

${ }^{1}$ Department of Dermatology, UT Southwestern Medical Center, Dallas, TX, USA; ${ }^{2}$ Arthritis and Clinical Research Immunology Program, Oklahoma Medical Research Foundation, Oklahoma City, OK, USA

Correspondence to: Benjamin Chong. Department of Dermatology, UT Southwestern Medical Center, 5323 Harry Hines Blvd. Dallas, TX 75390-9069, USA. Email: Ben.Chong@utsouthwestern.edu.

\begin{abstract}
Previous studies on blood transcription profiles of patients with cutaneous lupus (CLE) have primarily used a gene expression approach. The large amount of data involved in these studies can make data analysis and interpretation cumbersome. Consequently, a novel approach in gene expression analyses was devised that groups genes into transcriptional modules (i.e., apoptosis, protein synthesis, inflammation) to identify important themes and relevant genes. This approach was used to study gene expression in systemic lupus patients and subsequently identified a unique interferon signature. Thus, we aim to use the modular analysis approach to identify unique modules and genes of CLE patients in order to better understand their disease pathophysiology. We conducted RNA sequencing of whole transcriptomes of blood from 66 CLE patients and 10 human controls and subsequently performed modular analyses. Based on module scores, we performed an unsupervised cluster analysis to identify subgroups of CLE patients with distinct gene expression patterns. Statistical analyses comparing module scores of these clusters was performed using the Kruskal-Wallis tests with Bonferroni's correction. Modules were associated with
\end{abstract}

patient subgroups distinguished by demographic, clinical, and laboratory features such as CLE subtypes, autoantibody profiles (BioPlex ${ }^{\circledR} 2200$ ANA Screen with MDSS), and cytokine profiles (Thermo Scientific ProCarta xMAP cytokine assay kits). The unsupervised cluster analysis identified eight distinct clusters of CLE patients and controls. A neutrophilic predominant signature governed by azurophilic granule genes (i.e., BPI, LTF, MPO) and neutrophil chemotaxis genes (CEACAM6, CEACAM8) was seen in two groups of patients mostly with subacute lupus erythematosus (SCLE) [n=7 (77.8\% of total SCLE)] or lupus erythematosus tumidus (LET) [n=6 $(60 \%$ of total LET)] and European-American descent $[\mathrm{n}=28(53.6 \%$ of total European-Americans)]. Four groups consisting of a majority of discoid lupus erythematosus (DLE) patients $[\mathrm{n}=29(60 \%$ of total DLE)] had a predominant $\mathrm{T}$ cell signature driven by genes important for antigen presentation and $\mathrm{T}$ cell proliferation and differentiation (i.e., SKAP1, ITK). Our data suggests that unique cell populations may help differentiate CLE subgroups, which can potentially affect their disease course and treatment selection. Further studies examining individual gene and protein levels are necessary to confirm our hypotheses.

Keywords: Cutaneous lupus erythematosus (CLE); modular analysis; RNA sequencing

doi: 10.21037/atm.2021.AB006

Open Access Statement: This is an Open Access article distributed in accordance with the Creative Commons Attribution-NonCommercial-NoDerivs 4.0 International License (CC BY-NC-ND 4.0), which permits the noncommercial replication and distribution of the article with the strict proviso that no changes or edits are made and the original work is properly cited (including links to both the formal publication through the relevant DOI and the license). See: https://creativecommons.org/licenses/by-nc-nd/4.0/.

Cite this abstract as: Zhu J, Tran L, Zheng F, James J, Guthridge J, Chong B. A modular analysis approach to studying gene expression in cutaneous lupus erythematosus. Ann Transl Med 2021;9(5):AB006. doi: 10.21037/atm.2021.AB006 